\title{
How useful is uterine artery Doppler ultrasonography in predicting pre-eclampsia and intrauterine growth restriction?
}

\section{Lynne McLeod MD}

$\infty \quad$ See related article page 70 I

$\mathrm{P}$ re-eclampsia and intrauterine growth restriction remain important causes of maternal and neonatal complications and death. ${ }^{1-3}$ These 2 conditions are felt to be the result of abnormal placenta formation involving abnormal trophoblast invasion of spiral arteries and a reduction in vascular resistance in the uteroplacental circulation..$^{4-6}$ Although the incidence of pre-eclampsia in the general obstetric population is only $5 \%$, there is a potential for serious adverse outcomes. ${ }^{1,3,7,8}$ Maternal complications include the HELLP syndrome (hemolytic anemia, elevated liver enzymes, low platelet count), eclampsia, coagulopathy, stroke and death..$^{1,7,8}$ Newborns affected by intrauterine growth restriction are at increased risk for hypertension, * cardiovascular disease and diabetes later in life. ${ }^{9}$ Accurate prediction of pre-eclampsia and intrauterine growth restriction is therefore paramount to providing appropriate antenatal surveillance and therapy in an effort to improve perinatal outcomes.

The uteroplacental circulation can be assessed by means of Doppler ultrasonography of the uterine arteries. ${ }^{10}$ This

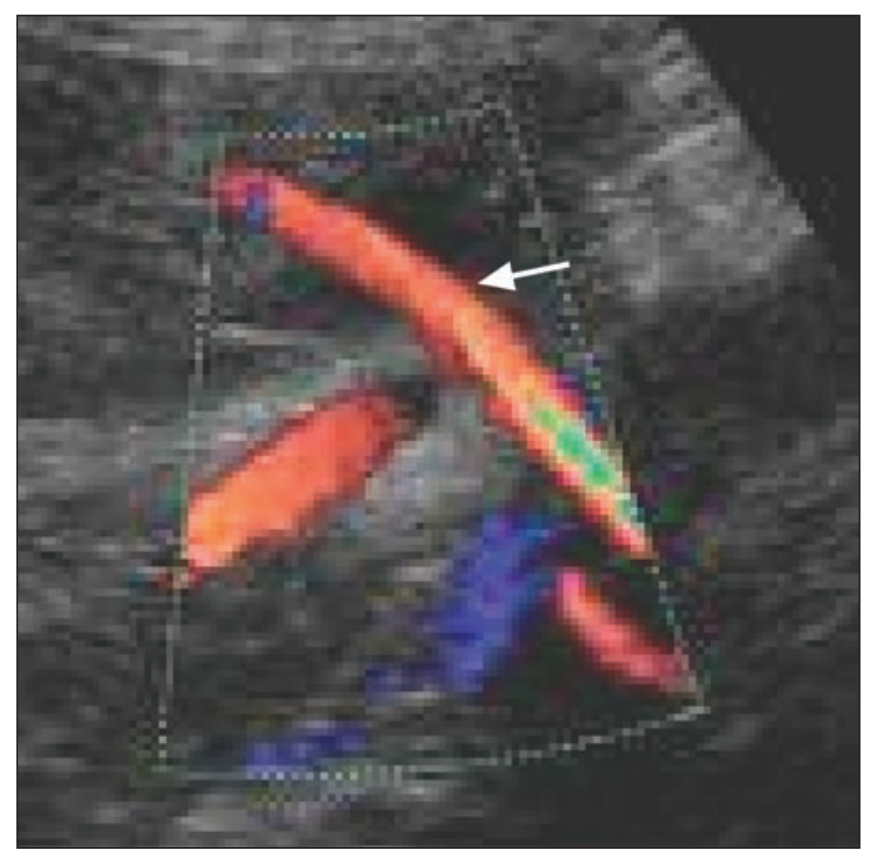

Figure 1: Colour Doppler ultrasound scan showing uterine artery (arrow).

\section{Key points of the article}

- Pre-eclampsia and intrauterine growth restriction are important causes of maternal and neonatal complications and death.

- Doppler ultrasonography of the uterine arteries is a promising technique for predicting these perinatal complications.

- More research is needed to determine the ideal timing of uterine artery Doppler ultrasonography and the ideal combination of this technology with other antenatal screening tests in an effort to improve perinatal outcomes.

procedure has been reported in numerous studies to be a promising technique for predicting the level of risk for preeclampsia and intrauterine growth restriction. ${ }^{11-15}$ In a normal pregnancy, impeded flow in the uterine artery decreases with increasing gestational age.

Uterine artery Doppler ultrasonography may be performed via the transvaginal or transabdominal route in the first or second trimester. Uterine artery waveforms are reported to be readily obtainable in more than $95 \%$ of patients. ${ }^{15}$ The uterine artery is identified with the use of colour Doppler ultrasonography (Figure I). Pulsed-wave Doppler ultrasonography is then used to obtain waveforms (Figure 2). Various indices can be calculated and assessed.

In this issue of $C M A J$, Cnossen and colleagues ${ }^{16}$ report the results of their systematic review and meta-analysis of studies in which Doppler assessment of the uterine arteries was used to predict pre-eclampsia and intrauterine growth restriction. Their review was extensive: they included 74 studies of preeclampsia and 6r of intrauterine growth restriction, with no language limitations; they evaluated the accuracy of 15 Doppler indices; and they used well-described and robust statistical methods for their analyses.

One of the most widely studied Doppler indices is the pulsatility index (calculated as the peak systolic flow minus the end diastolic flow divided by the mean flow). An increased pulsatility index has been associated with an increased risk for pre-eclampsia and intrauterine growth restriction..$^{13-15}$ The presence of an early diastolic notch in the waveform (Figure 3) has also been shown in several studies to be associated

Lynne McLeod is with the Department of Obstetrics and Gynecology, IWK Health Centre, Halifax, NS 
with adverse outcomes. The likelihood ratio, sensitivity and specificity for each Doppler index and specific outcome have varied among studies, but the predictive relationship for adverse outcomes has been consistently reported.

Cnossen and colleagues found that uterine artery Doppler ultrasonography more accurately predicted pre-eclampsia than intrauterine growth restriction and that the most powerful Doppler index for predicting pre-eclampsia was an increased pulsatility index with notching in the second trimester. For severe pre-eclampsia, they found that an increased pulsatility index or bilateral notching best predicted the condition. The authors' conclusions are in agreement with those from recent studies. ${ }^{17,18}$ With respect to intrauterine growth restriction, Cnossen and colleagues found that an increased pulsatility index alone or in combination with notching was most valuable for predicting intrauterine growth restriction in low-risk women, whereas an increased resistance index was the best predictor of the condition in high-risk women.

One of the challenges of conducting a review in this area is the variability in definitions of pre-eclampsia and intrauterine growth restriction, and in the causes. Maternal and perinatal outcomes are affected by the severity and duration of the condition as well as by preventive and therapeutic management strategies. These factors undoubtedly affect individual study results. In addition, the studies reviewed by Cnossen and colleagues were heterogeneous in their timing of Doppler assessment, severity of disease and outcomes, and inclusion of other screening modalities.

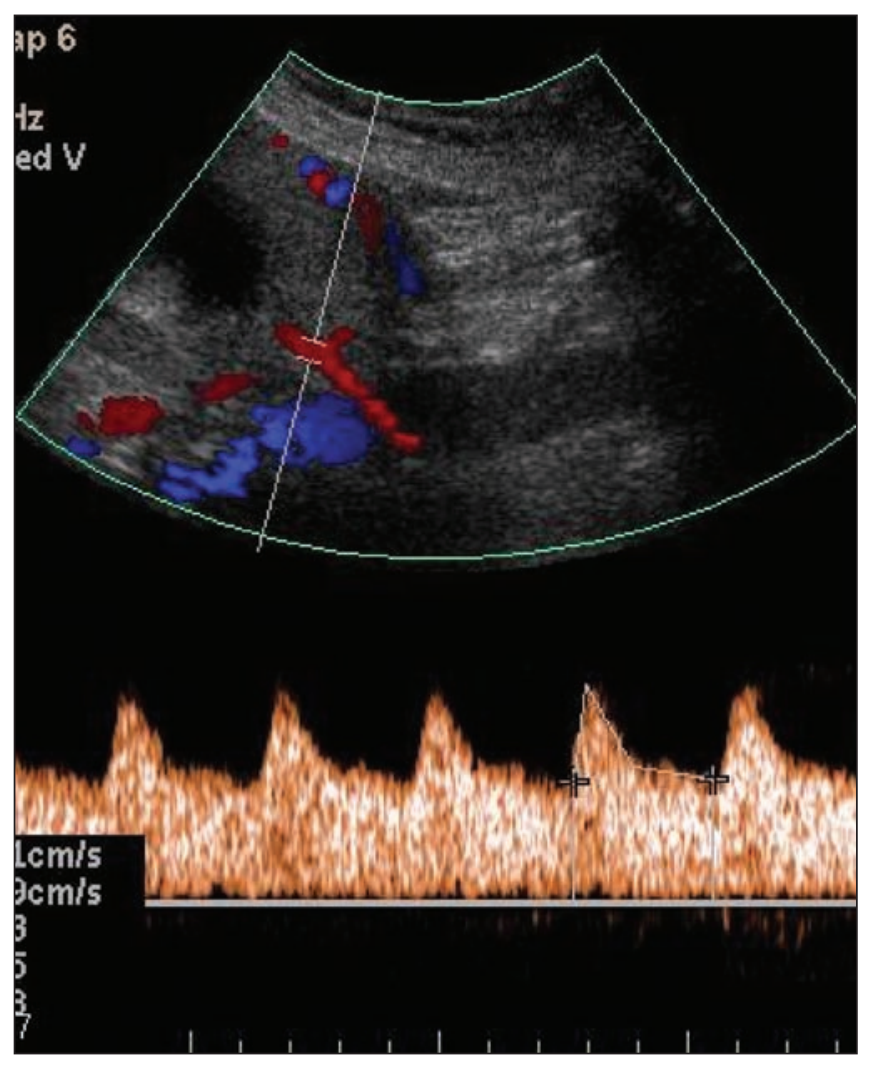

Figure 2: Uterine artery Doppler ultrasound scan showing normal waveform.
Although pre-eclampsia and intrauterine growth restriction are not uncommon and may pose serious health risks to both mother and fetus, we are still searching for preventive strategies for these at-risk pregnancies. The first step is to identify patients at risk, followed by increased surveillance and therapy. Uterine artery Doppler ultrasonography in isolation, and perhaps in combination with other modalities, may by the key to improved perinatal outcomes in these clinical conditions. In addition to uterine artery Doppler ultrasonography, a variety of proteins and hormones have been studied as potential early biomarkers of pre-eclampsia and intrauterine growth restriction. Second-trimester maternal serum screening markers for aneuploidy (the presence of an abnormal number of chromosomes), $\beta$ human chorionic gonadotropin ( $\beta$-hCG) and $\alpha$-fetoprotein have been associated with increased risk. ${ }^{19,20}$ Alterations in first-trimester serum levels of the biomarkers placental protein-I3 (PP-I3) and pregnancy-associated plasma protein-A (PAPP-A) have also been reported to be predictors of adverse outcomes. ${ }^{14,17,18,20}$ The purpose of the review by Cnossen and colleagues was not to address the potential additional benefit of measuring maternal biomarkers. However, consideration of a combination of screening tests in a select patient population may be beneficial to patients and health care resources.

The best screening tests have to be accessible, available and relatively inexpensive. They must also provide reproducible results and be acceptable to patients. Uterine artery Doppler ultrasonography fulfills these criteria and is relatively easy to perform with training and experience. According to Cnossen and colleagues' findings, this procedure could be done in conjunction with routine anatomy ultrasonography at I8-20 weeks' gestation. However, in some areas the availability of uterine artery Doppler ultrasonography may be limited to specialized obstetrics ultrasound units.

In conclusion, Cnossen and colleagues are to be commended for their thorough systematic review of the current literature on this important topic and for increasing our awareness of the potential benefits of uterine artery Doppler assessment. More research is needed to identify the ideal timing of screening with this technology and the ideal combina-

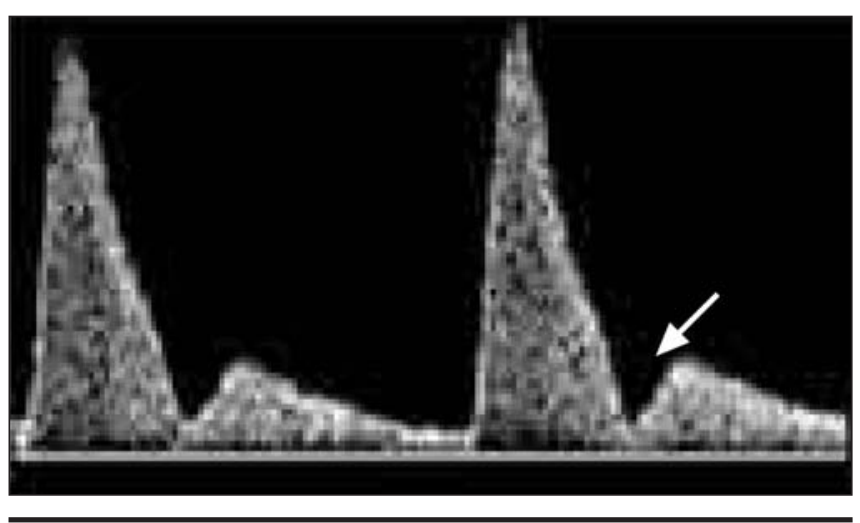

Figure 3: Uterine artery Doppler ultrasound scan showing early diastolic notch (arrow). The presence of diastolic notches is associated with an increased risk of pre-eclampsia and intrauterine growth restriction. 
tion of this technology with other predictors of pre-eclampsia and intrauterine growth restriction in an effort to improve perinatal outcomes. This would include identification of patient risk factors and antenatal screening - uterine artery Doppler ultrasonography and measurement of maternal serum biomarkers. The ideal combination would allow health care providers to screen the appropriate women and target antenatal surveillance and therapeutic interventions at those who were at risk for pregnancy complications in hopes of improving outcomes.

Competing interests: None declared.

\section{REFERENCES}

I. Sibai B, Dekker G, Kupferminc M. Pre-eclampsia. Lancet 2005;365:785-99.

2. Khan KS, Wojdyla D, Say L, et al. WHO analysis of causes of maternal death: a systematic review. Lancet 2006;367:1066-74.

3. Walker J. Pre-eclampsia. Lancet 2000;356:1260-5.

4. Khong TY, De Wolf F, Robertson WB, et al. Inadequate maternal vascular response to placentation in pregnancies complicated by pre-eclampsia and small for gestational age infants. BrJObstet Gynaecol I986;93:1049-59.

5. Pijnenborg R, Anthony J, Davey DA, et al. Placental bed spiral arteries in the hypertensive disorders of pregnancy. Br JObstet Gynaecol I99I;98:648-55.

6. Shanklin DR, Sibai BM. Ultrastructural aspects of pre-eclampsia. I:Placental bed and boundary vessels. Am J Obstet Gynecol I989; I6I:735-4I.

7. Wen SW, Huang L, Liston R, et al.; Maternal Health Study Group, Canadian Perinatal Surveillance System. Severe maternal morbidity in Canada, I99I-200I. CMAJ 2005;173:759-64.

8. Roberts JM, Cooper DW. Pathogenesis and genetics of pre-eclampsia. Lancet 200I;357:53-6.

9. Barker DJ, Osmond C, Golding J, et al. Growth in utero, blood pressure in childhood and adult life and mortality from cardiovascular disease. BMJ I989; 298:564-7.
Io. Campbell S, Pearce JMF, Hackett G, et al. Qualitative assessment of uteroplacental blood flow: Early screening test for high risk pregnancies. Obstet Gynecol I986; 68:649-53.

II. Martin AM, Bindra R, Curcio P, et al. Screening for pre-eclampsia and fetal growth restriction by uterine artery Doppler at II-I4 weeks of gestation. Ultrasound Obstet Gynecol 200I; 18:583-6.

I2. Gomez O, Martinez JM, Figueras F, et al. Uterine artery Doppler at II-I4 weeks of gestation to screen for hypertensive disorders and associated complications in an unselected population. Ultrasound Obstet Gynecol 2005;26:490-4.

13. Albaiges G, Missfelder-Lobos H, Lees C, et al. One-stage screening for pregnancy complications by color Doppler assessment of the uterine arteries at 23 weeks' gestation. Obstet Gynecol 2000;96:559-64.

I4. Spencer K, Yu CK, Cowans NJ, et al. Prediction of pregnancy complications by first trimester maternal serum PAPP-A and free beta-hCG and with second trimester uterine artery Doppler. Prenat Diagn 2005;25:949-53.

I5. Papageorghiou AT, Yu CKH, Bindra R, et al. Multicentre screening for pre-eclampsia and fetal growth restriction by transvaginal uterine artery Doppler at 23 weeks of gestation. Ultrasound Obstet Gynecol 200I;18:44I-9.

I6. Cnossen JS, Morris RK, ter Riet G, et al. Use of uterine artery Doppler ultrasonography to predict pre-eclampsia and intrauterine growth restriction: a systematic review and bivariable meta-analysis. $C M A J$ 2008;178:70I-II.

I7. Pilalis A, Souka P, Antsaklis P, et al. Screening for pre-eclampsia and fetal growth restriction by uterine artery Doppler and PAPP-A at II-I4 weeks' gestation. Ultrasound Obstet Gynecol 2007;29:135-40.

I8. Spencer K, Cowans NJ, Chefetz I, et al. First trimester maternal serum PP-I3, PAPP-A and second trimester uterine artery Doppler pulsatility index as markers of pre-eclampsia. Ultrasound Obstet Gynecol 2007;29:I28-34.

I9. Raty R, Koskinen P, Alanen A, et al. Prediction of pre-eclampsia with maternal midtrimester total renin, inhibin A, AFP, and free beta hCG levels. Prenat Diagn I999;I9:I22-7.

20. Yaron Y, Cherry M, Kramer RL. Second trimester maternal serum screening: maternal serum alpha fetoprotein, beta chorionic gonadotropin, estriol, and their various combinations as predictors of pregnancy outcome. Am J Obstet Gynecol I999;18I:968-74.

Correspondence to: Dr. Lynne McLeod, Department of Obstetrics and Gynecology, IWK Health Centre, 5850/5980 University Ave., PO Box 9700, Halifax NS B3K 6R8; fax 902 425-II25; lynne.mcleod@iwk.nshealth.ca

\section{CMAJ's Editorial Fellowship}

The CMAJ EDITORIAL FELLOwSHIP provides an exciting opportunity for physicians early in their training to discover the inner workings of a leading medical journal. Applications are invited from individuals who have completed their medical training and residency, as well as clinical epidemiology training, who are interested in obtaining a rich experience in medical writing, editing and publishing. The fellow participates in all aspects of journal production, ranging from selecting and editing manuscripts to soliciting commentaries and review articles. Fellows are also expected to write extensively and are encouraged to develop theme issues, series or other journal innovations.

The position is full time for one year and is based at CMAJ's offices in Ottawa. The salary is based on the equivalent residency or fellowship remuneration in Ontario.

The next round of applications is for the 2008 fellowship, which begins summer 2008. The application deadline is March 25, 2008.

For more information, please contact

Dr. Paul C Hébert, Editor-in-Chief, at paul.hebert@cma.ca.

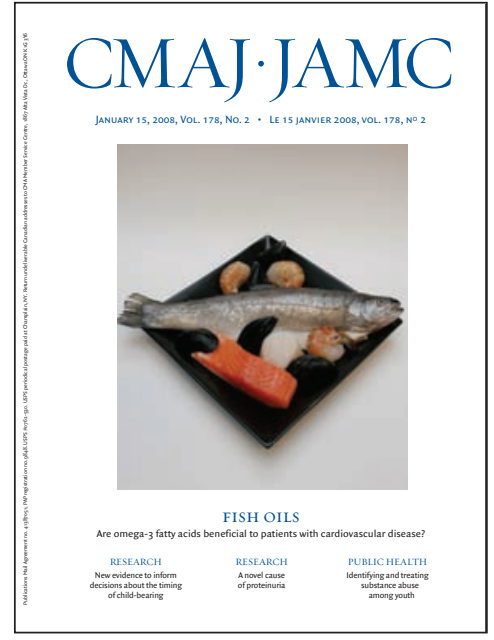

